\title{
Resurgence of Diphtheria in North Kerala, India, 2016: Laboratory Supported Case-Based Surveillance Outcomes
}

OPEN ACCESS

Edited by:

Marc Jean Struelens, European Centre for Disease Prevention and Control, Sweden

Reviewed by: Marisa Silvia Castro, Instituto de Estudios de la Inmunidad Humoral (CONICET-UBA), Argentina

Carlos Henrique Alencar Federal University of Ceará,

Brazil

${ }^{*}$ Correspondence:

Veeraraghavan Balaji vbalaji@cmcvellore.ac.in

Specialty section:

This article was submitted to Infectious Diseases Surveillance, Prevention and Treatment,

a section of the journal

Frontiers in Public Health

Received: 15 April 2017 Accepted: 04 August 2017 Published: 30 August 2017

Citation:

Sangal L, Joshi S, Anandan S, Balaji V, Johnson J, Satapathy A, Haldar P, Rayru R, Ramamurthy S, Raghavan A and Bhatnagar P (2017) Resurgence of Diphtheria in North Kerala, India, 2016: Laboratory Supported Case-Based Surveillance Outcomes.

Front. Public Health 5:218. doi: 10.3389/fpubh.2017.00218

\begin{abstract}
Lucky Sangal ${ }^{1}$, Sudhir Joshi ${ }^{1}$, Shalini Anandan ${ }^{2}$, Veeraraghavan Balaji ${ }^{2 *}$, Jaichand Johnson ${ }^{3}$, Asish Satapathy ${ }^{4}$, Pradeep Haldar ${ }^{5}$, Ramesh Rayru ${ }^{6}$, Srinath Ramamurthy ${ }^{7}$, Asha Raghavan ${ }^{8}$ and Pankaj Bhatnagar ${ }^{1}$
\end{abstract}

${ }^{1}$ WHO India, World Health Organization, New Delhi, India, ${ }^{2}$ Department of Clinical Microbiology, Christian Medical College, Vellore, India, ${ }^{3}$ State Public Health Laboratory, Thiruvananthapuram, India, ${ }^{4}$ WHO India, World Health Organization, Bangalore, India, ${ }^{5}$ Ministry of Health and Family Welfare, Government of India, New Delhi, India, ${ }^{6}$ Ministry of Health, Government of Kerala, Thiruvananthapuram, India, ${ }^{7}$ WHO India, World Health Organization, Kozhikode, India, ${ }^{8}$ WHO India, World Health Organization, Thiruvananthapuram, India

Introduction: As part of national program, laboratory supported vaccine preventable diseases surveillance was initiated in Kerala in 2015. Mechanisms have been strengthened for case investigation, reporting, and data management. Specimens collected and sent to state and reference laboratories for confirmation and molecular surveillance. The major objective of this study is to understand the epidemiological information generated through surveillance system and its utilization for action.

Methods: Surveillance data captured from reporting register, case investigation forms, and laboratory reports was analyzed. Cases were allotted unique ID and no personal identifying information was used for analysis. Throat swabs were collected from investigated cases as part of surveillance system. All Corynebacterium diphtheriae isolates were confirmed with standard biochemical tests, ELEK's test, and real-time PCR. Isolates were characterized using whole genome-based multi locus sequence typing method. Case investigation forms and laboratory results were recorded electronically. Public health response by government was also reviewed.

Results: A total of 533 cases were identified in 11 districts of Kerala in 2016, of which 92\% occurred in 3 districts of north Kerala; Malappuram, Kozhikode, and Kannur. Almost $79 \%$ cases occurred in $>10$ years age group. In $<18$ years age group, $62 \%$ were male while in $\geq 18$ years, $69 \%$ were females. In $<10$ years age group, $31 \%$ children had received three doses of diphtheria vaccine, whereas in $\geq 10$ years, $3 \%$ cases had received all doses. Fifteen toxigenic $C$. diphtheriae isolates represented 6 novel sequence types (STS) (ST-405, ST-408, ST-466, ST-468, ST-469, and ST-470). Other STs observed are ST-50, ST-295, and ST-377.

Conclusion: Diphtheria being an emerging pathogen, establishing quality surveillance for providing real-time information on disease occurrence and mortality is imperative. The epidemiological data thus generated was used for targeted interventions and to 
formulate vaccine policies. The data on molecular surveillance have given an insight on strain variation and transmission patterns.

Keywords: vaccine preventable diseases, Corynebacterium diphtheriae, outbreak, molecular surveillance, multilocus sequence typing

\section{INTRODUCTION}

The Universal Immunization Program (UIP) aims to reduce morbidity and mortality from vaccine preventable diseases (VPD) to levels that no longer constitute a public health problem. Laboratory supported VPD surveillance is an important tool for providing real-time information on the occurrence of VPDs and measures the progress made by the immunization program (1).

The state of Kerala launched laboratory supported case-based VPD surveillance, including diphtheria, with support from World Health Organization-National Polio Surveillance Project (WHO-NPSP) in April 2015. The suspected cases are reported by reporting network that includes both public and private sector health facilities. Mechanisms have been strengthened for case investigation, reporting, and data management. This system has been utilized for effective case management and public health interventions in response to identified cases and outbreaks of diphtheria.

Laboratory confirmation of clinically suspected diphtheria cases is crucial for accurately classifying cases and build confidence in the surveillance system. As part of the national program, capacity building and system strengthening has been done for two identified laboratories in Kerala state for performing laboratory diagnosis of diphtheria as per WHO norms. These are State Public Health Laboratory (SPHL), Thiruvananthapuram, and Microbiology department at Government Medical College, Kozhikode. A national reference laboratory responsible for standardization of laboratory procedures, technology transfer to other network laboratories, and quality assurance has been established at Christian Medical College (CMC), Vellore.

Numerous reports on the outbreak assessment and epidemiological surveillance of diphtheria from different parts of India have been previously reported (2-5). However, most of these reports are of data generated by various academic institutions as short-term research projects. The main objective of this study is to understand the epidemiological information generated through the surveillance system and how best it was utilized to take evidence-based public health measures. To the best of our knowledge, this is the first report on the diphtheria outbreak in Kerala along with molecular surveillance.

\section{MATERIALS AND METHODS}

We analyzed surveillance data available from the VPD surveillance system in the state of Kerala. The data sources were reporting register, case investigation forms, and laboratory reports.

As per standard VPD surveillance system protocol, cases were reported through a network of reporting sites. The case definition that was used for diphtheria surveillance was the standard WHO definition.

An illness of upper respiratory tract characterized by the following:

- Laryngitis or pharyngitis or tonsillitis,

- and adherent membranes of tonsils, pharynx, and/or nose.

All suspected cases that were reported to the system were investigated preferably within $48 \mathrm{~h}$ by trained medical officers. A unique identification number was allotted at district level to all such cases. The investigating officer or a trained health worker collected a throat swab specimen and send it to a WHO supported laboratory for culture and toxigenicity test.

The case investigation forms were recorded electronically by WHO-NPSP in software called Surveillance Information Management System that could be assessed real time at local, state, and national levels. The system recorded multiple variables like socio-demographic data, clinical history, treatment history, vaccination status, contact information, travel history, and type of sample taken.

The laboratory results were captured in software called Vaccine Preventable Disease Laboratory Information for Action and fed forwarded to national level. The case-based records and laboratory results were then electronically merged by an automated process based on unique identification number of the cases.

Identification of any diphtheria case in Kerala was followed by active case search in the community and contact tracing. Reporting of high number of diphtheria cases in the state had alerted the state government of Kerala for taking focused actions to curtail the further spread of diphtheria outbreaks. A steering committee, State Technical Advisory Group on Immunization (STAGI), was constituted to oversee the response and make appropriate recommendations. Other organizations of the private sector like Indian Medical Association, Indian Academy of Pediatrics, and religious heads were involved to spread awareness and early notification of diphtheria suspected cases. The cases were managed and public health interventions were taken as outlined in the VPD surveillance guidelines. We have reviewed the response of the government to diphtheria cases and recommendations of the steering committee.

This study was based on surveillance data available from national program and molecular typing of Corynebacterium diphtheriae isolates from routine specimen collection. It does not involve any participation of patients; hence, informed consent was not required. The study was in agreement with Government of India and State Government of Kerala. This study was approved by Institutional Research Board of CMC, Vellore at the meeting conducted on 28-10-2016 (IRB Min No: 9706). 


\section{Laboratory Methods for Identification of Diphtheria \\ Culture}

The throat swabs were processed and the isolate was identified by culture using $5 \%$ sheep blood agar and Serum Tellurite agar. C. diphtheriae colonies were confirmed with the Cystinase test growing black colonies with brown halo on Tinsdale agar (DIFCO, USA). The species was further confirmed with biochemical testing based on the utilization of glucose, dextrose, sucrose, maltose sugars followed by nitrate and urease tests (Fisher Scientific, MA, USA).

\section{Toxigenicity Testing-Modified Elek's Test}

The toxigenicity testing was performed according to the previously reported method (6). Briefly, Elek's agar medium with $20 \%$ newborn bovine serum was used to plate two test strains and three control strains on one plate. An antitoxin strip (500 U/ ml, VINS Bioproducts Ltd., Hyderabad, India) was placed on the center of the plate and incubated at $37^{\circ} \mathrm{C}$ for 24 and $48 \mathrm{~h}$. At $24 \mathrm{~h}$ using a suitable light source plates were observed for precipitin lines of identity between the test strains and the strong and weak positive control strains.

\section{Real-time PCR Detection}

DNA was extracted using QIAamp DNA blood mini kit (QIAGEN, Germany). The target genes include $r p o B$ for $C$. diphtheriae, $C$. ulcerans, and toxA gene fragment of $C$. diphtheriae. Primers and conditions used in this study were previously reported for the Light Cycler (Roche, USA) PCR platform (Table 1) (7). This study tailored the similar protocol using the 7,500 Fast Real-time PCR (Applied Bio Systems, USA). Briefly, the PCR reaction set-up included $95^{\circ} \mathrm{C}$ for $10 \mathrm{~min}$; followed by 45 cycles of $95^{\circ} \mathrm{C}$ for $15 \mathrm{~s}$, and $60^{\circ} \mathrm{C}$ for $30 \mathrm{~s}$. C. diphtheriae Ct cut off values for positivity were minimum 31.24 and maximum 34.06 , for C. ulcerans minimum 28.96 and maximum 31.12, and for toxA minimum 31.05 and maximum 35.03 .

\begin{tabular}{|c|c|c|c|}
\hline Target & $\begin{array}{l}\text { Primer/ } \\
\text { probe }\end{array}$ & Sequence $5^{\prime}-3^{\prime}$ & $\begin{array}{l}\text { Amplicon } \\
\text { length } \\
\text { (bp) }\end{array}$ \\
\hline $\begin{array}{l}\text { C. } \\
\text { diphtheriae }\end{array}$ & dip_rpobF & CGT TCG CAA AGA TTA CGG AAC CA & 97 \\
\hline rpoB & $\begin{array}{l}\text { dip_rpobR } \\
\text { dip_probe }\end{array}$ & $\begin{array}{l}\text { CAC TCA GGC GTA CCA ATC AAC } \\
\text { HEXd-AGG TTC CGG GGC TTC TCG } \\
\text { ATA TTC A-BHQ1 }\end{array}$ & \\
\hline $\begin{array}{l}\text { C. ulcerans } \\
\text { rpoB }\end{array}$ & $\begin{array}{l}\text { ulc_rpobF } \\
\text { ulc_rpobR } \\
\text { ulc_probe }\end{array}$ & $\begin{array}{l}\text { TTC GCA TGG CTC ATT GGC AC } \\
\text { TCC AGG ATG TCT TCC AGT CC } \\
\text { FAM-CCA GCA GGA GGA GCT GGG } \\
\text { TGA A-BHQe1 }\end{array}$ & 98 \\
\hline Toxin & $\begin{array}{l}\text { toxAF } \\
\text { toxAR } \\
\text { diptoxHP }\end{array}$ & $\begin{array}{l}\text { CTT TTC TTC GTA CCA CGG GAC TAA } \\
\text { CTA TAA AAC CCT TTC CAA TCA TCG } \\
\text { TC } \\
\text { ROX-AAG GTA TAC AAA AGC CAA AAT } \\
\text { CTG GTA CAC-BHQ2 }\end{array}$ & 117 \\
\hline
\end{tabular}

The primer sequences used were previously reported (7).

\section{Whole Genome Sequencing-Based Multi-Locus Sequence Typing (MLST)}

Twenty-one lab confirmed isolates were subjected to whole genome sequencing by Ion torrent next generation sequencing technology as described in our previous study (8). Briefly, the whole genomic DNA was extracted as described before, and the purified DNA was used for the preparation of library fragments using the Ion Plus fragment library kit (Ion Torrent; Life Technologies, USA). Whole-genome sequencing was performed using the Ion Torrent PGM sequencer (Thermo Scientific Fisher Corp., USA) using 400-bp chemistry. The Raw sequence reads were assembled using SPAdes 5.0.0.0 tool embedded in Ion torrent server.

Multi-locus sequence typing profile of the isolates was predicted from the whole genome sequences by MLST 1.8 tool of the Centre for Genomic Epidemiology server (9). The allele loci of the unknown sequence types (STs) were submitted to PUBMLST database. Clonal analysis of the identified STs was performed by eBURST software $(10,11)$. The concatenated seven housekeeping gene sequences were aligned by Clustal-W and the phylogenetic tree was constructed using the Neighbor Joining algorithm by MEGA software. The evolutionary distances were computed by Jukes-Cantor method.

\section{RESULTS}

After initiating the VPD surveillance in Kerala, 533 cases have been identified in 11 districts in 2016. A surge in reporting of suspected Diphtheria cases was noticed from May 2016 onward and a total number of 527 cases occurred in time period of 31 May 2016 to 30 November 2016. Figures 1 and 2 show the time and place distribution of these 527 cases. The first case, reported on 31 May, was from Tanur block of Malappuram district. Soon after the reporting of diphtheria cases increased in northern part of Kerala with maximum number of cases from Malappuram district $(n=229)$ followed by Kozhikode $(n=190)$, Kannur $(n=64)$, Wayanad $(n=16)$, Palakkad $(n=15)$, Thrissur $(n=4)$, Kasargod $(n=3)$. Six cases were reported from three districts of South Kerala, two each from Alappuzha, Ernakulam, and Thiruvananthapuram. Figure 2 shows the epi curve of Diphtheria cases for the three districts, Malappuram, Kozhikode, and Kannur, which have the highest number of cases. The typical bell-shaped curve is noticed for Malappuram and Kozhikode almost starting, reaching peak, and waning at the same time. The cases in Kannur started 3-4 weeks later and show multiple peaks in the same time duration.

Table 2 shows the age and sex distribution of 527 diphtheria cases. Most of the cases (79\%) occurred in more than 10 years age group. Just $7 \%$ cases were children under 5 years while another $7 \%$ were in the age group of more than 45 years. The overall sex distribution of diphtheria cases is almost proportionate in males and females. However, in preschool and school going age group $(0-18$ years) males seem to be more affected $(62 \%)$ and among all the male cases $71 \%$ are in this age group. In adult population ( $\geq 18$ years) females were more affected (69\%) and among all females $60 \%$ were in this age group.

The religion break up of diphtheria cases shows that $69 \%$ of total cases occurred in Muslims. The proportions of cases in 


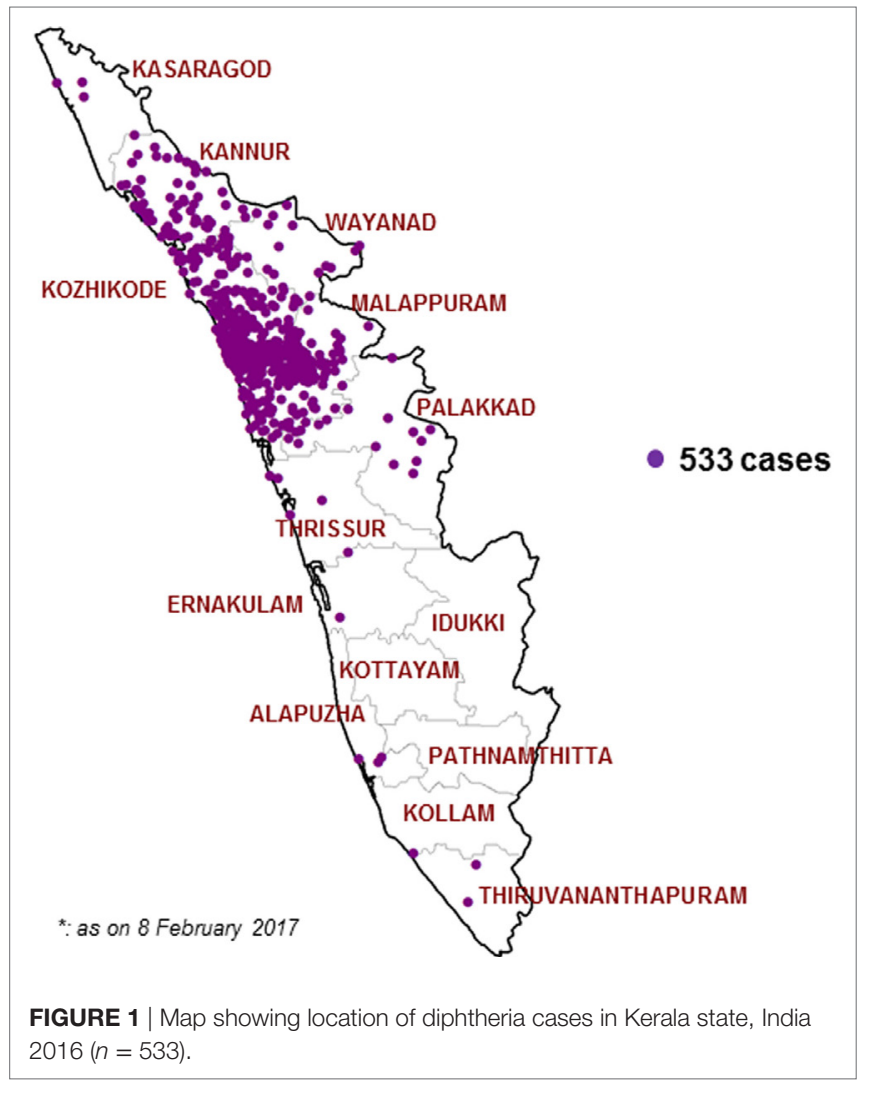

Muslim community in Malappuram, Kozhikode, and Kannur are 83,61 , and $52 \%$, respectively. Two cases of age 15 and 16 years died in Malappuram district of which one was laboratory confirmed Diphtheria $(\mathrm{CFR}=0.4 \%)$.

We analyzed immunization with diphtheria containing vaccine in different age groups (Table 3 ). As per UIP schedule in India diphtheria antigen is given at 6,10 , and 14 weeks with two boosters at 16-24 months and 5-6 years of age. In children less than 5 years and in the age group of 5-10 years, 23 and $11 \%$ children, respectively, were found to be vaccinated against diphtheria as per age. In children less than 10 years, $31 \%$ children had received three doses of primary vaccination and $68 \%$ were either not vaccinated or their vaccination status was not known. In age group of above 10 years, $3 \%$ cases received fives doses of diphtheria containing vaccine and $7 \%$ were partially immunized.

Response to diphtheria cases: sudden increase of cases from week 22 onward, death of index case, and media reports put the state government at high alert. A total of approximately 575 480 doses of $\mathrm{Td}$ vaccine was procured by the state and stocked at Malappuram district for distribution to neighboring districts. Erythromycin prophylaxis was provided to close contacts as evident from action taken reports.

\section{Laboratory Identification of Diphtheria}

The number of cases in relation to the positivity for the samples received from the different centers is represented in the Table 4. MLST analysis for 21 C. diphtheriae isolates from SPHL,

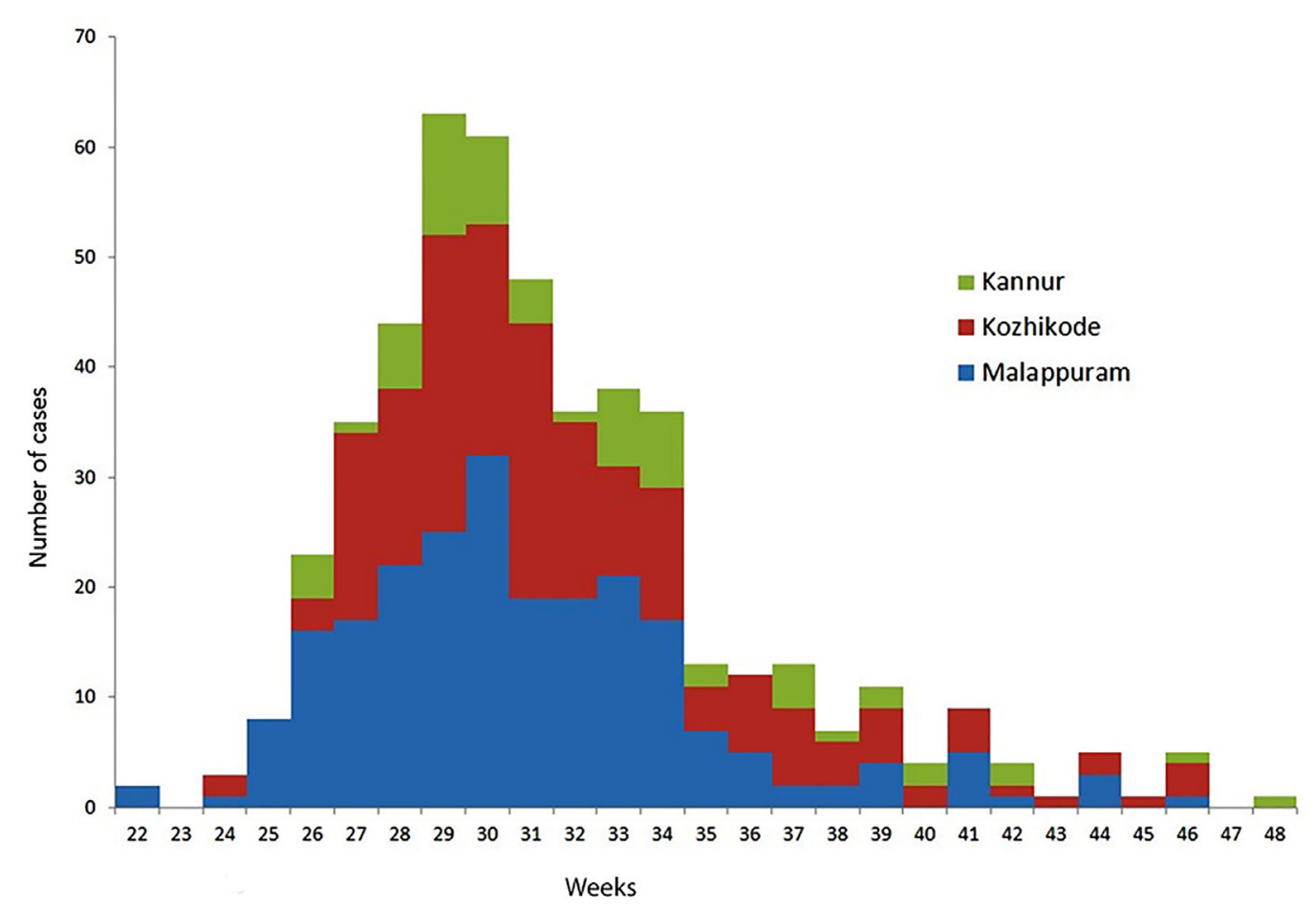

FIGURE 2 | Epidemic curve of diphtheria cases in Malappuram, Kozhikode, and Kannur districts of Kerala, weeks 22-48; 2016 ( $n=483)$. 
Trivandrum revealed diversity among the STs. Nine STs ST-50, ST-295, ST-377, ST-405, ST-408, ST-466, ST-468, ST-469, and ST-470 were seen in the isolates, with majority of them belong to the new STs, ST-405 $(n=7)$ and ST-466 $(n=4)$, respectively. The STs ST-50, ST-295, ST-408, ST-468, ST-469, and ST-470 were seen in one isolate each and two isolates belonging to the unknown ST's were submitted for inclusion in the C. diphtheriae PubMLST database.

The phylogeny tree was constructed by neighbor joining algorithm for the MLST pattern derived from the allele loci of seven housekeeping genes obtained from the Kerala isolates and reference strains (NCTC 05011, HCO1, HC04, C7 beta ${ }^{\text {tox+ }}$, NCTC 13129, INCA402) (Figure 3). The isolates WD1472, WD58, and WD 62 was closely related to the reference strains HC01 (ST128), HC04 (ST-175), and PWD (ST-44). The PW8 strain was widely used for the production of diphtheria toxoid vaccine. The C. diphtheriae isolate WD 70 with unknown ST was closely related to the toxigenic strain C7 beta tox with ST ST-26. The six isolates with the new ST ST-405 was related to NCTC toxigenic strain 13129 (ST-8), which was isolated from the 2-year-old female with clinical diphtheria in United Kingdom. The NCTC 05011 strain originating from United Kingdom was found related to the isolates with STs ST-468, ST-50, and ST-405, respectively. The clonal relationship between the isolates from Kerala and other parts of the world is represented by eBURST diagram (Figure 4).

TABLE 2 | Age and sex distribution of diphtheria cases in Kerala, India (weeks 20-48).

\begin{tabular}{lccc}
\hline Age group (in years) & Females & Males & Total \\
\hline $0-5$ & 12 & 23 & 35 \\
$5-10$ & 39 & 54 & 93 \\
$10-18$ & 58 & 103 & 161 \\
$18-45$ & 135 & 63 & 198 \\
$\geq 45$ & 29 & 10 & 39 \\
Total & 273 & 253 & 526 \\
\hline
\end{tabular}

TABLE 3 | Vaccination status of diphtheria cases in Kerala, India (weeks 22-48) 2016 ( $n=527)$.

\begin{tabular}{lrrrrrrr}
\hline \multicolumn{7}{c}{ Doses of diphtheria containing vaccine } \\
\hline Age groups & $\mathbf{0}^{\mathbf{a}}$ & $\mathbf{1}$ & $\mathbf{2}$ & $\mathbf{3}$ & $\mathbf{4}$ & $\mathbf{5}$ & Total \\
\hline $0-5$ & 24 & 0 & 0 & 4 & 7 & 0 & 35 \\
$5-10$ & 63 & 1 & 1 & 4 & 15 & 10 & 94 \\
$10-18$ & 135 & 7 & 3 & 4 & 4 & 8 & 161 \\
$18-45$ & 192 & 0 & 1 & 1 & 2 & 2 & 198 \\
$>45$ & 39 & & & & & & 39 \\
\hline
\end{tabular}

${ }^{a}$ Cases with unknown immunization history were included as zero doses.
eBURST analysis revealed the ST-50 was associated with the second major clonal complex (26 ST's) with the founder ST-5. The newly reported ST in India, ST-405 forms complex with STs 305 and 308, respectively. The STs 295, 377, ST-408, ST-466, ST-469, and ST-470 do not belong to any clonal complexes circulating in world.

\section{DISCUSSION}

Of the total diphtheria cases reported in Kerala in 2016, 92\% occurred in three districts of north Kerala; Malappuram, Kozhikode, and Kannur. The outbreak in this area could be due to the existence of pockets of low immunization coverage. Media reports have shown that vaccine acceptance is an issue noticed in certain sections of the community in north Kerala $(12,13)$.

The epi curve, from 22 to 48 weeks, shows that soon after the first case in Malappuram, the neighboring districts of Kozhikode and Kannur were largely affected. The epi curve of Kozhikode almost overlaps that of Malappuram suggesting that it was a part of the same event. The undulating curve of Kannur may be due to multiple exposures of susceptible population in this district over a time period of 25-43 weeks.

The surveillance data clearly indicates the shift in burden of diphtheria cases in adolescents and adults, as also noticed in other recently occurred Diphtheria outbreaks globally $(14,15)$. This could be due to traditional low immunization coverage in these pockets or waning of immunity after natural infection or any previous vaccination. Opportunities to provide immunization services to protect adolescents and adults should be encouraged, such as at school leaving age, pregnancy, etc. Tetanus toxoid (TT) vaccination is already a part of immunization schedule for adolescents (10 and 16 years of age) and pregnant women. Replacing TT vaccination with $\mathrm{Td}$ (TT and low dose diphtheria toxoid) vaccine has the potential to provide protection against diphtheria without any additional burden to the immunization program (16).

The sex distribution of cases shows that males are more affected during childhood or school going age group. However, after the age of 18 years, the females are significantly more affected. As women most commonly work in households and as caregivers in domestic settings, the chances of their exposure is high (14).

The religion break up of diphtheria cases shows that most of them are in Muslim community (69\%). The Muslim population in Malappuram is high (70\%) compared with rest of Kerala (27\%), it is 39\% in Kozhikode, and 29\% in Kannur as per 2011 census. However, it is noticed that the proportion of diphtheria cases among Muslims are higher than the population proportion of Muslims in these three districts. It is 83, 61, and 52\% in Malappuram, Kozhikode, and Kannur, respectively.

TABLE 4 | Laboratory confirmation of $C$. diphtheriae isolates by culture, ELEK's test, and real-time PCR for rpoB and tox- $A$ gene.

\begin{tabular}{|c|c|c|c|c|c|c|}
\hline \multirow{2}{*}{$\begin{array}{l}\text { Centers } \\
2016\end{array}$} & \multirow[t]{2}{*}{ Specimen received } & \multirow[t]{2}{*}{ Isolate received } & \multirow[t]{2}{*}{ Culture positives } & \multirow[t]{2}{*}{ Elek's test positives } & \multicolumn{2}{|c|}{ Real-time PCR results } \\
\hline & & & & & C. diphtheriae (rpoB) & toxA \\
\hline Thiruvananthapuram, State & Isolate & 21 & 20 & 18 & 20 & 18 \\
\hline Public Health Laboratory & Throat Swab & 69 & 1 & 1 & 2 & 2 \\
\hline Calicut, ASTER MIMS & Isolate & 1 & 1 & 1 & 1 & 1 \\
\hline
\end{tabular}


Kerala witnessed large number of diphtheria cases in 2016 despite traditionally achieving DTP3 coverage above $85 \%$ (87\% in 2007-2008 and 93\% in 2012-2013) $(17,18)$. The three districts

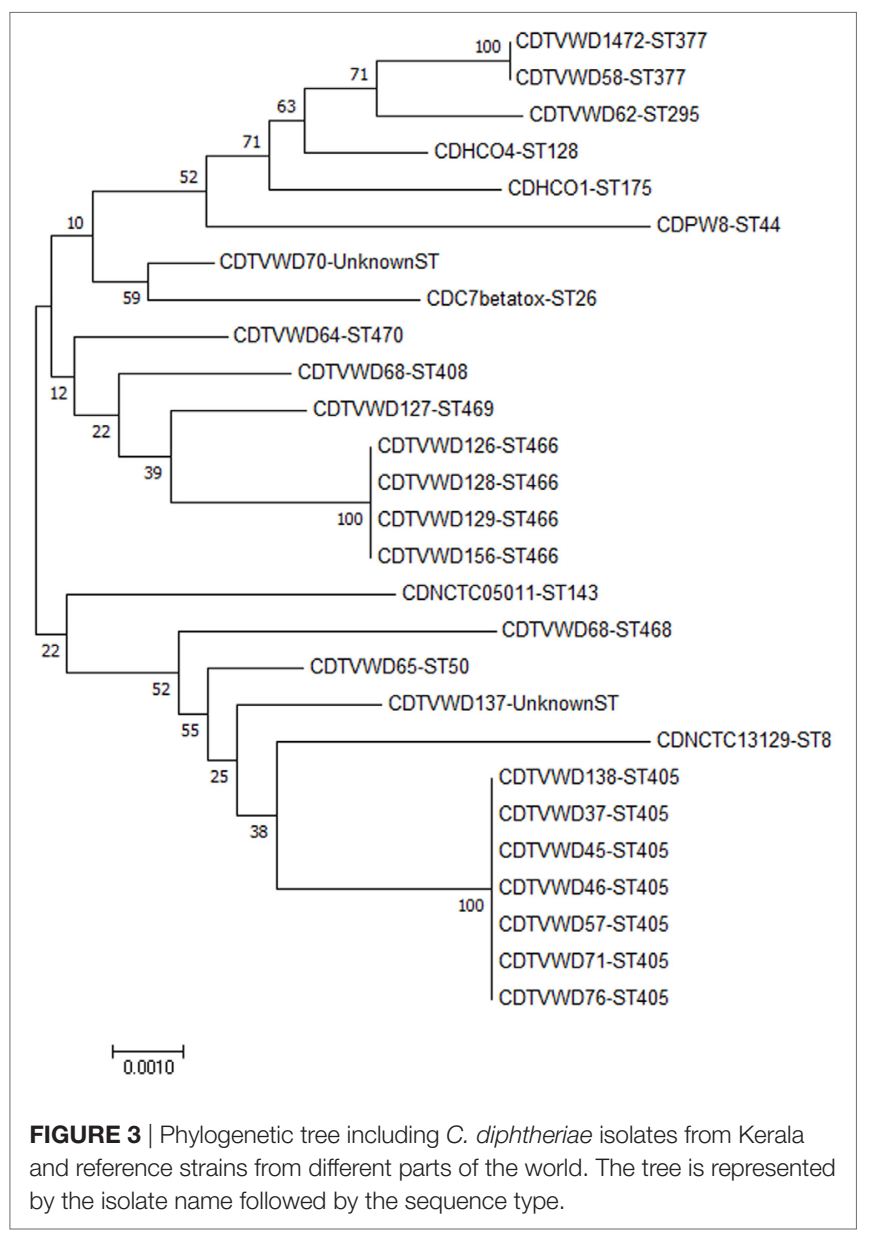

of Kerala viz; Malappuram, Kozhikode, and Kannur that had witnessed large number of diphtheria cases in 2016 also had DTP3 coverage of 89,97 , and 95\%, respectively, as per DLHS4. Certain sections of Muslim community in this area are reluctant to give vaccination to their children $(12,13)$ which might have led to pockets of susceptible cohorts. The government should target interventions in increasing vaccine acceptance among Muslims through involvement of religious leaders, community influencers, and educational institutions. These interventions should include booster doses because it has been seen that booster dose coverages fall leading to outbreaks (14). The polio eradication initiative in India has already proved that targeted interventions to increase vaccine acceptance in resistant communities reaps long term benefits to immunization program (19).

The early identification of diphtheria occurrence and death of two cases by the VPD surveillance system alerted the government to mount an early response. Constitution of STAGI and participation of both public/private sector effectively took appropriate and timely actions, like chemoprophylaxis and $\mathrm{Td}$ vaccination, to curtail the further spread of diphtheria in the community. Involvement of Department of Education was instrumental for Td vaccination in schools. The contact tracing at households and schools led to the early detection and management of additional cases resulting in low mortality and morbidity due to diphtheria. Diphtheria usually has a case fatality rate in the range of 5-10\% (20) however, higher CFRs have been documented in many parts of the world. A diphtheria outbreak reported in Assam in 2015 documented 20\% CFR (21).

The outbreak of diphtheria has been associated with concomitant periods of increase in the genomic diversity of the organism. The detailed investigation on the genomic information of the C. diphtheriae following the outbreak will help in analyzing the genetic diversity and transmission dynamics of the potential genes involved in virulence and development of resistance to antimicrobials. In recent years, advancement in next generation

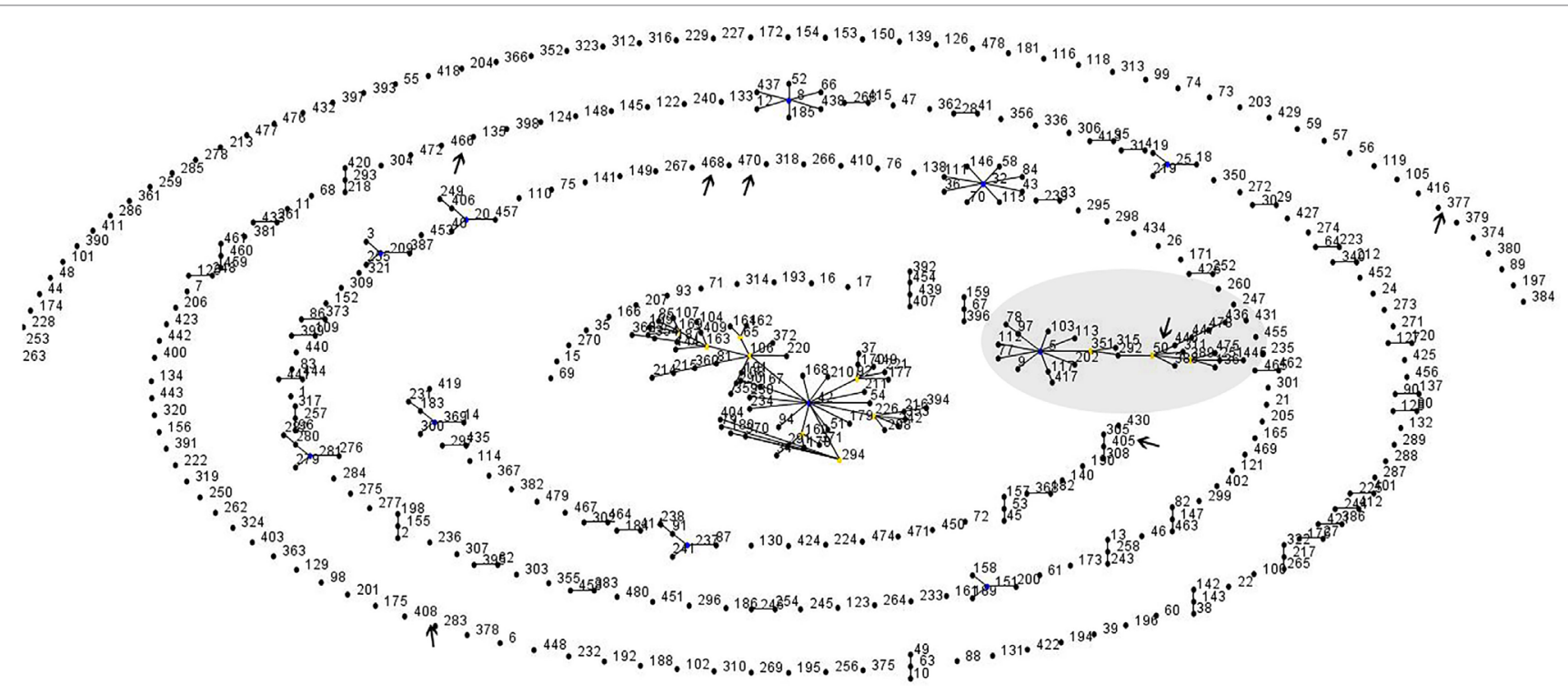

FIGURE 4 | Global clonal relationship of the $C$. diphtheriae isolates predicted by eBURST analysis. Sequenced types observed in the Kerala outbreak were marked. 
sequencing technologies has enabled fast and detailed investigation of the organism and help in identifying the clonality and transmission of the strain, involved in the infection. The application of appropriate typing methods is essential not only in outbreak investigations to monitor the evolution and spread of epidemic clones of $C$. diphtheriae but also in understanding and predicting epidemics.

The toxigenic strains sequenced in this study were characterized using traditional phenotypic and whole genome-based MLST typing methods. Seven of the toxigenic $C$. diphtheriae isolates (WD37, WD45, WD 46, WD57, WD71, WD76, and WD138) sequenced in this study represents the novel ST, ST-405, which was reported in our previous study isolates from Kadapa region of Andhra Pradesh (8). This implicates that same ST is circulating in two different states of South India (Andhra Pradesh and Kerala). The phylogram suggests the existence and persistence of a lineage that has been evolving and recurring in India, with special mention to the different districts in the state of Kerala. Two of the predicted STs (ST-405 and ST-50) associated with the outbreak in Kerala was related to clonal complexes circulating in the world, whereas the other STs are unique to the region.

\section{CONCLUSION}

Laboratory supported VPD surveillance is an important tool for providing real-time information on the occurrence of VPDs and to take immediate actions to curtail the spread and reduce mortality. The surveillance data generated the evidence on changing epidemiology of diphtheria that helps the program not only to take targeted interventions but also to formulate vaccine policies. The current study in Kerala highlights the need for strengthening laboratory supported VPD surveillance across country. Further, the data on molecular surveillance of Diphtheria generated through laboratory support has given an insight on strain variation and transmission patterns. Further studies on the

\section{REFERENCES}

1. John TJ, Samuel R, Balraj V, John R. Disease surveillance at district level: a model for developing countries. Lancet (1998) 352(9121):58-61. doi:10.1016/ S0140-6736(97)10494-9

2. Allam RR, Uthappa CK, Duerst R, Sorley E, Udaragudi PR, Kampa $\mathrm{S}$, et al. A case-control study of diphtheria in the high incidence city of Hyderabad, India. Pediatr Infect Dis (2016) 35(3):253-6. doi:10.1097/ INF.0000000000001034

3. Meera M, Rajarao M. Diphtheria in Andhra Pradesh - a clinical-epidemiological study. Int J Infect Dis (2014) 19:74-8. doi:10.1016/j. ijid.2013.10.017

4. Dandinarasaiah M, Vikram BK, Krishnamurthy N, Chetan A, Jain A. Diphtheria re-emergence: problems faced by developing countries. Indian J Otolaryngol Head Neck Surg (2013) 65(4):314-8. doi:10.1007/ s12070-012-0518-5

5. Phalkey RK, Bhosale RV, Joshi AP, Wakchoure SS, Tambe MP, Awate P, et al. Preventing the preventable through effective surveillance: the case of diphtheria in a rural district of Maharashtra, India. BMC Public Health (2013) 13(1):317. doi:10.1186/1471-2458-13-317

6. Engler KH, Glushkevich T, Mazurova IK, George RC, Efstratiou A. A modified Elek test for detection of toxigenic corynebacteria in the diagnostic laboratory. J Clin Microbiol (1997) 35(2):495-8. mechanisms of invasion, transmission dynamics of the disease, strain variation, and population structure of $C$. diphtheriae is necessary. Diphtheria being the emerging pathogen of increasing significance worldwide, it is imperative to take appropriate measures to control the disease.

\section{INFORMED CONSENT AND ETHICS}

Since the study includes the surveillance and molecular typing of $C$. diphtheriae isolates, it does not involve participation of the patients. Hence, informed consent is not required. This study was approved by Institutional Research Board of Christian Medical College, Vellore at the meeting conducted on 28-10-2016 (IRB Min No: 9706).

\section{AUTHOR NOTE}

In any use of this article, there should be no suggestion that $\mathrm{WHO}$ endorses any specific organization, products, or services. The use of the WHO logo is not permitted. This notice should be preserved along with the article's original URL. The authors alone are responsible for the views expressed in this article and they do not necessarily represent the views, decisions, or policies of the institutions with which they are affiliated.

\section{AUTHOR CONTRIBUTIONS}

LS, SJ, SA, and VB conceived, designed the study. JJ, AS, SR, and $\mathrm{AR}$ conducted the surveillance. $\mathrm{PH}, \mathrm{RR}$, and $\mathrm{PB}$ organized and validated the study outcomes. LS, SJ, SA, and VB involved in computational analysis and drafting the manuscript. $\mathrm{PH}, \mathrm{RR}$, and $\mathrm{PB}$ reviewed and finalized the manuscript.

\section{FUNDING}

Government of India and World Health Organization, 2016.

7. De Zoysa A, Efstratiou A, Mann G, Harrison TG, Fry NK. Development, validation and implementation of a quadruplex real-time PCR assay for identification of potentially toxigenic corynebacteria. J Med Microbiol (2016) 65(12):1521-7. doi:10.1099/jmm.0.000382

8. Veeraraghavan B, Anandan S, Sekar SKR, Gopi R, Ragupathi NKD, Ramesh $\mathrm{S}$, et al. First report on the draft genome sequences of Corynebacterium diphtheriae isolates from India. Genome Announc (2016) 4(6):e1316-1316. doi:10.1128/genomeA.01316-16

9. Larsen MV, Cosentino S, Rasmussen S, Friis C, Hasman H, Marvig RL, et al. Multilocus sequence typing of total genome sequenced bacteria. J Clin Microbiol (2012) 50(4):1355-61. doi:10.1128/JCM.06094-11

10. Feil EJ, Li BC, Aanensen DM, Hanage WP, Spratt BG. eBURST: inferring patterns of evolutionary descent among clusters of related bacterial genotypes from multilocus sequence typing data. J Bacteriol (2004) 186(5):1518-30. doi:10.1128/JB.186.5.1518-1530.2004

11. Spratt BG, Hanage WP, Li B, Aanensen DM, Feil EJ. Displaying the relatedness among isolates of bacterial species-the eBURST approach. FEMS Microbiol Lett (2004) 241(2):129-34. doi:10.1016/j.femsle.2004.11.015

12. Rajeev GR. The fallacies of the faithful. Available from: http://www.thehindu. com/search/?q=14479789

13. Herriman R. India: Diphtheria takes the life of unvaccinated Kerala teen. (2016). Available from: http://outbreaknewstoday.com/india-diphtheriatakes-the-life-of-unvaccinated-kerala-teen-59420/ 
14. Garib Z, Danovaro-Holliday M, Tavarez Y, Leal I, Pedreira C. Diphtheria in the Dominican Republic: reduction of cases following a large outbreak. Rev Panam Salud Pública (2015) 38(4):292-9.

15. Wagner K, White J, Lucenko I, Mercer D, Crowcroft N, Neal S, et al. Diphtheria in the postepidemic period, Europe, 2000-2009. Emerg Infect Dis (2012) 18(12):217-25. doi:10.3201/eid1802.110987

16. Murhekar M, Bitragunta S. Persistence of diphtheria in India. Indian J Community Med (2011) 36(2):164. doi:10.4103/0970-0218.84141

17. District Level Household and Facility Survey-3, State Fact Sheet Kerala 2007-08. International Institute for Population Sciences. Available from: http://rchiips. org/pdf/rch3/report/KL.pdf

18. District Level Household and Facility Survey-4, State Fact Sheet Kerala 2012-13. International Institute for Population Sciences. Available from: http://rchiips. org/pdf/dlhs4/report/KE.pdf

19. Weiss WM, Rahman M, Solomon R, Singh V, Ward D. Outcomes of polio eradication activities in Uttar Pradesh, India: the Social Mobilization Network (SM Net) and Core Group Polio Project (CGPP). BMC Infect Dis (2011) 11(1):117. doi:10.1186/1471-2334-11-117
20. Hamborsky J, Kroger A. Epidemiology and prevention of vaccine-preventable diseases. E-Book: The Pink Book. Public Health Foundation (2015).

21. DaS PP, Patgiri SJ, Saikia L, PauL D. Recent outbreaks of diphtheria in Dibrugarh district, Assam, India. J Clin Diagn Res (2016) 10(7):DR01-03. doi:10.7860/JCDR/2016/20212.8144

Conflict of Interest Statement: The authors declare that the research was conducted in the absence of any commercial or financial relationships that could be construed as a potential conflict of interest.

Copyright (C) 2017 Sangal, Joshi, Anandan, Balaji, Johnson, Satapathy, Haldar, Rayru, Ramamurthy, Raghavan and Bhatnagar. This is an open-access article distributed under the terms of the Creative Commons Attribution License (CC BY). The use, distribution or reproduction in other forums is permitted, provided the original author(s) or licensor are credited and that the original publication in this journal is cited, in accordance with accepted academic practice. No use, distribution or reproduction is permitted which does not comply with these terms. 